\title{
Radiation Dose and Prognosis of Ultra-Low-Dose Stress-First Myocardial Perfusion SPECT in Patients with Chest Pain Using a High-Efficiency Camera
}

\author{
Andrew J. Einstein ${ }^{1,2}$, Lynne L. Johnson ${ }^{1}$, Albert J. DeLuca ${ }^{1}$, Andrew C. Kontak ${ }^{1}$, Daniel W. Groves ${ }^{1}$, Jennifer Stant ${ }^{1}$, \\ Ted Pozniakoff ${ }^{1}$, Bin Cheng ${ }^{3}$, LeRoy E. Rabbani ${ }^{1}$, and Sabahat Bokhari ${ }^{1}$ \\ ${ }^{I}$ Cardiology Division, Department of Medicine, Columbia University Medical Center and New York-Presbyterian Hospital, New York, \\ New York; ${ }^{2}$ Department of Radiology, Columbia University Medical Center and New York-Presbyterian Hospital, New York, \\ New York; and ${ }^{3}$ Department of Biostatistics, Columbia University, New York, New York
}

\begin{abstract}
Although SPECT myocardial perfusion imaging (MPI) provides valuable information about patients with chest pain, there is growing concern regarding its radiation burden and lengthy duration. New high-efficiency (HE) cameras and stress-first protocols both offer the potential to markedly reduce radiation. No previous study has assessed outcomes and radiation doses of patients undergoing MPI on an HE-SPECT camera using an ultra-low-dose stress-first protocol. Methods: One hundred patients presenting to the emergency department with chest pain who were candidates for stress-first MPI underwent injection of approximately $185 \mathrm{MBq}(5 \mathrm{mCi})$ of $99 \mathrm{mTc}-$ tetrofosmin at peak stress, followed by supine and prone imaging on an HE-SPECT camera. Same-day rest imaging was performed on patients with any abnormality on imaging after stress. Radiation effective dose was calculated from administered and residual activities. Patients were contacted 3 mo after discharge, and electronic records were accessed to evaluate the need for reevaluation for chest pain, additional imaging, or cardiac events. Results: Stress-only imaging was performed in 69 patients, for whom radiation effective dose averaged $0.99 \mathrm{mSv}$ and study duration, $117 \mathrm{~min}$. Radiation dose averaged $2.22 \mathrm{mSv}$ over all patients. At $3 \mathrm{mo}, 96$ patients were free of major adverse cardiac events, repeat hospital chest pain evaluation, and repeat imaging or stress testing. One year after MPI and hospital discharge, all patients were living and without acute coronary syndrome. Conclusion: HE-SPECT stress-only imaging can be performed in more than two thirds of chest pain patients without a high pretest probability of a stress perfusion defect, with excellent prognosis, a radiation dose averaging $1 \mathrm{mSv}$, and a test duration of less than $2 \mathrm{~h}$.
\end{abstract}

Key Words: SPECT; stress-first; stress-only; high-efficiency camera; radiation dose reduction; myocardial perfusion imaging

J Nucl Med 2015; 56:545-551

DOI: 10.2967/jnumed.114.150664

A

pproximately 6 million patients each year present to U.S. emergency departments with chest pain (1), and an additional 2 million with other symptoms that may be consistent with myocar-

Received Oct. 29, 2014; revision accepted Jan. 30, 2015.

For correspondence or reprints contact: Andrew J. Einstein, Columbia University Medical Center, 622 W. 168th St., PH 10-203A, New York, NY 10032. E-mail: andrew.einstein@columbia.edu

Published online Mar. 5, 2015.

COPYRIGHT (C) 2015 by the Society of Nuclear Medicine and Molecular Imaging, Inc. dial ischemia (2). For patients in whom there is a reasonable possibility that their symptoms could be caused by acute coronary syndrome, professional society recommendations include a functional study to exclude inducible ischemia $(2,3)$. The most commonly performed such test is SPECT myocardial perfusion imaging (MPI). The information provided by MPI can diagnose coronary disease with high sensitivity, stratify risk, guide patient management, and control costs. However, there is growing concern regarding the radiation burden of MPI. Its radiation effective dose averages $10 \mathrm{mSv}$, equivalent to 500 posteroanterior chest radiographs (4), and can be as high as $27 \mathrm{mSv}$ for some dual-isotope studies (5). Moreover, a SPECT MPI examination can be a lengthy procedure, requiring some patients to be present in the nuclear cardiology laboratory for more than $4 \mathrm{~h}$, delaying discharge and thereby leading to dissatisfaction from many patients and health care providers. These concerns may have contributed in some practice settings to the decreased use of MPI ( 6 ) and the substitution of other testing modalities such as stress echocardiography or coronary CT angiography.

Conventional Anger SPECT (A-SPECT) cameras are based on a design advanced by Berkeley electrical engineer Hal Anger in 1957. Contemporary A-SPECT cameras typically have 2 large thallium-doped sodium-iodide $(\mathrm{NaI}(\mathrm{Tl}))$ crystal detectors that are coupled to arrays of photomultiplier vacuum tubes and are used with low-energy, high-resolution collimators. These traditional cameras detect less than $0.02 \%$ of photons (7). In contrast, new high-efficiency (HE) cameras detect more photons by virtue of incorporating up to 19 solid-state cadmium-zinc-telluride detectors arrayed around the patient, collimator geometry designed to optimize photon detection, and advanced reconstruction algorithms. Presently, 2 such HE cameras (8) are available for clinical practice, offering the potential to reduce administered activity and thus radiation dose to patients, while still maintaining image quality. One recent study of 101 patients demonstrated that a single ultra-lowdose (ULD) rest injection of a radiopharmaceutical, with a radiation dose averaging $1.15 \mathrm{mSv}$ and imaged on an HE-SPECT camera, was associated with superior image quality to standard low-dose imaging using an A-SPECT camera, with similar total perfusion deficit, summed rest score, and ejection fraction (9). However, no previous study has assessed clinical outcomes of patients who were imaged using stress-first or stress-only ULD HE-SPECT as part of their emergency department inpatient evaluation for chest pain. We hypothesized that using ULD HE-SPECT stress-first imaging would be an excellent, clinically useful functional study to exclude 
inducible ischemia, resulting in more than $90 \%$ of patients free, at 3 mo after MPI and hospital discharge, of the composite endpoint of death, nonfatal myocardial infarction, unstable angina, repeat emergency department visit for chest pain evaluation, or additional stress testing or functional or anatomic cardiac imaging. Additionally, we hypothesized that this clinical utility would be achieved at a low radiation dose of $1 \mathrm{mSv}$ for stress-only studies, low rates of rest imaging, and decreased time requirement for MPI.

\section{MATERIALS AND METHODS}

\section{Patient Population}

We prospectively enrolled patients who initially presented with chest pain to the emergency department at Columbia University Medical Center/New York-Presbyterian Hospital, which provides health care to a multiethnic urban population, and who were deemed to be candidates to undergo stress-first MPI. Chest pain at our institution is classified into 4 categories, in which level 1 constitutes ST-elevation myocardial infarction; level 2, probable unstable angina or non-STelevation myocardial infarction; level 3, chest pain without an alternative explanation but normal or nondiagnostic electrocardiograms and at least 2 negative serum troponin levels taken $6 \mathrm{~h}$ or more apart; and level 4, chest pain believed not to be of cardiac origin. Per institutional clinical pathways for acute coronary syndrome and chest pain $(10,11)$, MPI is performed solely in patients with level 3 chest pain during the hospitalization. Thus, level 3 chest pain, the provider's planning to perform MPI, the attending nuclear cardiologist's consideration of stress-first MPI on an HE-SPECT camera as a clinically appropriate protocol for the patient, the patient's ability and willingness to provide informed consent, and age $18 \mathrm{y}$ or older were required for inclusion in the study. Patients could not be included if they were a poor candidate for stress-only imaging. This excluded patients who had previous MPI with evidence of scar, previous MPI with evidence of ischemia and no subsequent revascularization, a high pretest probability of a perfusion defect on MPI, or a known dilated left ventricle or cardiomyopathy; in all of these cases, rest imaging is required. Other exclusion criteria were body mass index (BMI) greater than $35 \mathrm{~kg} / \mathrm{m}^{2}$ and prisoner status. The Columbia University institutional review board approved this study, and all subjects gave written informed consent.

\section{Imaging Protocol}

As part of their clinical care, all patients underwent exercise or pharmacologic stress testing with $0.4 \mathrm{mg}$ of regadenoson in an intravenous push over $10-20 \mathrm{~s}$, or $140 \mu \mathrm{g} / \mathrm{kg} / \mathrm{min}$ of adenosine over 6 min. A 185-MBq (5-mCi) unit dose of ${ }^{99 \mathrm{~m}} \mathrm{Tc}$-tetrofosmin (Myoview; GE Healthcare) was calibrated and administered according to the exercise or pharmacologic stress testing protocol. The stress-first MPI was performed with the patient in the supine and prone positions, 30-60 min after administration, using 15-min acquisitions on an HE Discovery NM 530c camera with Alcyone technology (GE Healthcare), incorporating 19 cadmium-zinc-telluride detectors, pinhole collimation, stationary data acquisition, and 3-dimensional iterative reconstruction. Images after stress testing were reconstructed on a workstation (Xeleris II; GE Healthcare) and reviewed by an attending nuclear cardiologist. Patients with normal or probably normal perfusion on supine or prone imaging, normal left ventricular size $(<120 \mathrm{~mL}$ for men, $<92 \mathrm{~mL}$ for women), normal left ventricular function (ejection fraction $>45 \%$ ), and no abnormal right ventricular tracer uptake did not undergo rest imaging but rather were discharged with outpatient follow-up. Patients with an abnormality in any of the preceding factors received a second injection at rest approximately $3 \mathrm{~h}$ after the initial injection, using a dose equal to approximately 3 times the stress received activity plus 1.5 times the stress residual activity. Approximately 45-60 min later, they underwent supine rest imaging with a 15-min acquisition. Study duration was determined as the time from the initial calibration of activity, before stress testing, to the time the last imaging study (stress or rest) was completed. All studies were interpreted by an attending nuclear cardiologist, and clinical management based on the MPI was coordinated by the referring practitioner.

\section{Administered Activity and Estimation of Radiation Effective Dose}

Administered activity was measured using a dose calibrator (CRC15R; Capintec, Inc.) just before administration of the ULD injection, and residual activity in the syringe or needle was measured afterward. Received activity was calculated as the difference between administered and residual activities, each corrected for ${ }^{99 \mathrm{~m}} \mathrm{Tc}$-tetrofosmin decay. Similarly, measured administered activity, measured residual activity, and calculated received activity were determined for rest injections, if performed. Effective dose of radiation was estimated from received activities using a conversion factor based on the most recent ${ }^{99 \mathrm{~m}} \mathrm{Tc}-$ tetrofosmin dosimetry (12).

\section{Follow-up}

All patients were contacted 3 mo after MPI and hospital discharge to undergo a structured, scripted interview and to evaluate the need for reevaluation, such as repeat emergency department visit or hospitalization for chest pain; for reimaging with additional stress testing or functional or anatomic cardiac imaging (including angiography with or without revascularization); and for major adverse events including death, nonfatal myocardial infarction, and unstable angina. Additionally, the hospital's longitudinal electronic health record was accessed for all patients to evaluate events, procedures performed, and all episodes of care within 3 mo and within 12 mo after MPI and hospital discharge. Finally, the Social Security Death Index was queried more than $18 \mathrm{mo}$ after MPI and discharge to determine whether the patient had died within 12 mo of discharge and was therefore lost to follow-up.

\section{Statistical Analysis}

Continuous variables are described by mean \pm SD or median and range and compared using Student $t$ tests. Categoric variables are summarized as count and percentage and compared using $\chi^{2}$ tests. The primary, prespecified endpoint was freedom at 3 mo after MPI and hospital discharge of the composite endpoint of death, nonfatal myocardial infarction, unstable angina, repeat emergency department visit for chest pain evaluation, or repeat stress testing or functional or anatomic cardiac imaging. The primary, prespecified hypothesis was that freedom at 3 mo of the composite endpoint occurred in at least $90 \%$ of cases; this was assessed using an exact binomial test. Secondary analyses assessed events at 3 and $12 \mathrm{mo}$. Statistical analysis was performed using Stata/SE 13.1 (StataCorp) and R, version 3.0.1.

\section{Role of the Funding Source}

The study was conceived and performed by the principal and senior investigators. The decision as to the final study design remained solely that of the principal and senior investigators, with input from other investigators. At no point did the funding source have access to clinical or imaging data. The funding source played no role in the decision to publish or the content of the publication, and remained masked to the results of the study until immediately before its submission for publication.

\section{RESULTS}

\section{Patients and Doses}

Characteristics of the 100 patients enrolled are summarized in Table 1. Stress-only imaging was able to be performed in $69(69 \%)$ studies. Activities and radiation effective doses are summarized in 
TABLE 1

Patient Characteristics

\begin{tabular}{|c|c|c|c|c|}
\hline Characteristic & All & Stress-only & Stress and rest & $P$ \\
\hline Total subjects & 100 & 69 & 31 & \\
\hline Age (y) & $53.4 \pm 11.3$ & $52.9 \pm 8.7$ & $54.5 \pm 10.4$ & 0.21 \\
\hline Women & $60(60)$ & $44(44)$ & $16(16)$ & 0.25 \\
\hline Race/ethnicity & & & & 0.086 \\
\hline African-American & $21(21)$ & $12(17)$ & 9 (29) & \\
\hline Asian & $5(5)$ & $4(6)$ & $1(3)$ & \\
\hline Caucasian & $17(17)$ & $8(12)$ & $9(29)$ & \\
\hline Hispanic & $53(53)$ & $42(61)$ & $11(35)$ & \\
\hline Other & $4(4)$ & $3(4)$ & $1(3)$ & \\
\hline \multicolumn{5}{|l|}{ BMI } \\
\hline $\mathrm{kg} / \mathrm{m}^{2}$ & $27.9 \pm 3.4$ & $27.5 \pm 3.4$ & $28.8 \pm 3.3$ & 0.043 \\
\hline Range & $18.9-34.8$ & $18.9-34.8$ & $19.5-33.8$ & \\
\hline Diabetes mellitus & $15(15)$ & $9(13)$ & $6(19)$ & 0.66 \\
\hline Hypertension & $53(53)$ & $37(54)$ & $16(52)$ & 0.85 \\
\hline Hyperlipidemia & $40(40)$ & $23(33)$ & $17(55)$ & 0.042 \\
\hline Current smoking & $11(11)$ & $8(12)$ & $3(10)$ & 0.78 \\
\hline Family history of premature heart disease & $31(31)$ & $20(29)$ & $11(35)$ & 0.52 \\
\hline No risk factors & $15(15)$ & $13(19)$ & $2(6)$ & 0.11 \\
\hline Prior myocardial infarction & 0 & 0 & 0 & \\
\hline Stress type & & & & 1.00 \\
\hline Exercise, Bruce protocol & $84(84)$ & $58(84)$ & $26(84)$ & \\
\hline Adenosine & $3(3)$ & $2(3)$ & $1(3)$ & \\
\hline Regadenoson & $13(13)$ & $9(13)$ & $4(13)$ & \\
\hline
\end{tabular}

Qualitative data are expressed as numbers, followed by percentages in parentheses; continuous data are expressed as mean \pm SD.

Table 2 and Figure 1. The measured administered activity for stress imaging averaged $194 \mathrm{MBq}(5.23 \mathrm{mCi})$, and the calculated received activity, after correction for residual activity in the syringe and for decay, was $143 \mathrm{MBq}$ (3.87 mCi). The effective dose for a complete study, with stress imaging and rest imaging as needed, averaged $2.22 \mathrm{mSv}$, and the $69 \%$ of studies that were performed as stressonly imaging without the need for rest imaging averaged an effective dose of $0.99 \mathrm{mSv}$.

\section{Study Duration}

For stress-only imaging, study duration averaged less than $2 \mathrm{~h}$ (mean, $117 \pm 23 \mathrm{~min}$; median, $115 \mathrm{~min}$; range, 75-173 min). For studies requiring rest imaging, study duration was typically $4 \mathrm{~h}$ (mean, $241 \pm 44$ min; median, 234 min; range, 193-428 min). All supine and prone imaging was between 14 and 16 min in duration.

\section{Imaging Findings}

Sixty-nine patients had normal stress imaging and did not undergo rest imaging. Of the 31 patients undergoing both stress and rest imaging, 18 ultimately were clarified as having normal perfusion with a summed stress score (SSS) of 0. For the other 13 patients, 6 had SSS and summed rest score (SRS) of 1, 1 had SSS of 1 and SRS of 0,1 had SSS of 2 and SRS of 0, 4 had SSS between 3 and 6 with SRS also between 3 and 6 , and 1 had severe ischemia with SSS of 25 . In addition, among the 18 patients with normal perfusion were 2 patients with left ventricular systolic dysfunction and 3 with a dilated left ventricle.

\section{Patient Outcomes}

Ninety-six of the 100 patients $(96 \%)$ met the primary outcome criteria, at 3 mo after MPI and hospital discharge, of freedom from death, nonfatal myocardial infarction, unstable angina, repeat emergency department visit for chest pain evaluation, or repeat stress testing or functional or anatomic cardiac imaging. The associated exact $95 \%$ confidence interval for the percentage of patients meeting the primary outcome criteria was $90 \%$ to $99 \%$, and the corresponding $P$ value was 0.024 for the primary hypothesis that at least $90 \%$ of patients would be free of untoward events or need for repeat evaluation. This remained significant $(P=$ 0.027 ) when excluding the 2 patients for whom measured administered activity (178 and $184 \mathrm{MBq}$ ) was less than $185 \mathrm{MBq}$, although within $5 \%$ of the physician's prescribed activity of 185 MBq. Fourteen patients either were not able to be contacted or elected not to participate in the follow-up interview; 9 of these were seen in follow-up clinical visits after at least 3 mo with no report of these untoward events or repeat evaluation within 3 mo, and all 14 were living $1 \mathrm{y}$ after MPI and hospital discharge as per the Social Security Death Index. For the 4 patients not meeting the primary outcome criteria, one had a completely normal MPI result but nonetheless underwent an outpatient stress cardiac MR study, 
TABLE 2

Activities (MBq) and Radiation Effective Doses (mSv)

\begin{tabular}{|c|c|c|c|}
\hline Type of Study & Mean & SD & Range \\
\hline \multicolumn{4}{|l|}{ Stress-only studies $(n=69)$} \\
\hline \multicolumn{4}{|l|}{ Stress injection } \\
\hline Administered activity, measured & 193 & 7.4 & $178-214$ \\
\hline Residual activity, measured & 41 & 25 & $12-118$ \\
\hline Received activity, calculated & 143 & 26 & $61-178$ \\
\hline Effective dose, received & 0.99 & 0.18 & $0.42-1.23$ \\
\hline \multicolumn{4}{|l|}{ Stress-rest studies $(n=31)$} \\
\hline \multicolumn{4}{|l|}{ Stress injection } \\
\hline Administered activity, measured & 194 & 8.0 & $187-222$ \\
\hline Residual activity, measured & 44 & 25 & $6-102$ \\
\hline Received activity, calculated & 141 & 27 & $69-178$ \\
\hline Effective dose, received & 0.97 & 0.19 & $0.48-1.23$ \\
\hline \multicolumn{4}{|l|}{ Rest injection } \\
\hline Administered activity, measured & 609 & 94 & $462-832$ \\
\hline Residual activity, measured & 96 & 40 & $20-179$ \\
\hline Received activity, calculated & 499 & 102 & $321-732$ \\
\hline Effective dose, received & 3.99 & 0.82 & $2.57-5.86$ \\
\hline \multicolumn{4}{|l|}{ Total injection } \\
\hline Received activity & 640 & 100 & $433-841$ \\
\hline Effective dose, received & 4.96 & 0.80 & $3.34-6.56$ \\
\hline
\end{tabular}

whereas 3 presented again to the emergency department with similar symptoms, were discharged from the emergency department without hospital admission or repeat cardiac imaging, and were alive $1 \mathrm{y}$ later with no evidence of a repeat event. At $1 \mathrm{y}$ after MPI and hospital discharge, no patients had documentation of nonfatal myocardial infarction or unstable angina, whereas 5 additional patients had a repeat emergency department visit for chest pain evaluation or repeat stress testing or functional or anatomic cardiac imaging. All patients were living $1 \mathrm{y}$ after MPI and hospital discharge.

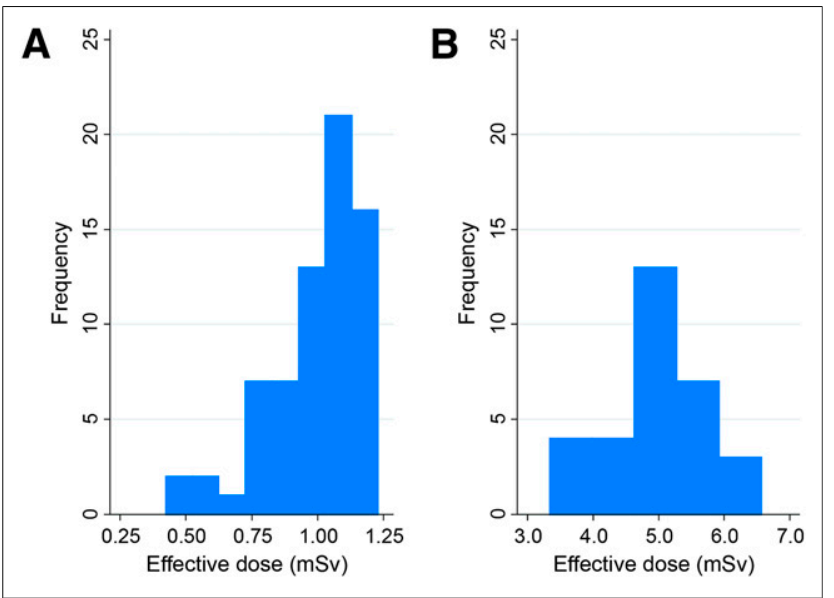

FIGURE 1. Effective doses received from myocardial perfusion imaging. (A) Stress-only studies. (B) Stress-rest studies.

\section{DISCUSSION}

Both the development of HE-SPECT cameras and the validation of stress-first/stress-only protocols (13) offer the potential to markedly reduce radiation dose from MPI. Their combination makes possible the performance of a complete nuclear stress test using ${ }^{99 \mathrm{~m}} \mathrm{Tc}$-tetrofosmin at a radiation dose of $1 \mathrm{mSv}$ or less (Fig. 2) and identification of ischemia with a single-day protocol at a radiation dose under $5 \mathrm{mSv}$ (Fig. 3). However, clinical outcomes in patients scanned with such a stress-first ULD HE-SPECT protocol have not been previously studied.

Moreover, combined supine and prone HE-SPECT imaging has been demonstrated to significantly reduce artifactual false-positive perfusion defects in comparison with supine-only imaging (14), particularly inferolaterally, and thus facilitates the performance of stress-only imaging in a greater proportion of patients. We observed that in a population of chest pain patients without a high pretest probability of a perfusion defect, ULD HE-SPECT MPI with both supine and prone imaging could be performed using a single injection in more than two thirds of patients, for whom the study length averaged less than $2 \mathrm{~h}$ and radiation dose averaged just under $1 \mathrm{mSv}$. It is important that prognosis was excellent, with only $4 \%$ of patients requiring additional cardiac imaging or presenting again to the hospital for chest pain evaluation within $3 \mathrm{mo}$, and no deaths or acute coronary syndromes at $1 \mathrm{y}$. Thus, stress-first ULD MPI using an HE-SPECT camera represents a potentially important approach to reduce radiation dose and length of stay, while still ensuring safe discharge, in patients presenting to the hospital with chest pain.

Our findings complement and build on a few previous studies that have evaluated HE-SPECT using a ${ }^{99 \mathrm{~m}} \mathrm{Tc}$ protocol with reduced activity. For example, the MILLISIEVERT study (9) compared 


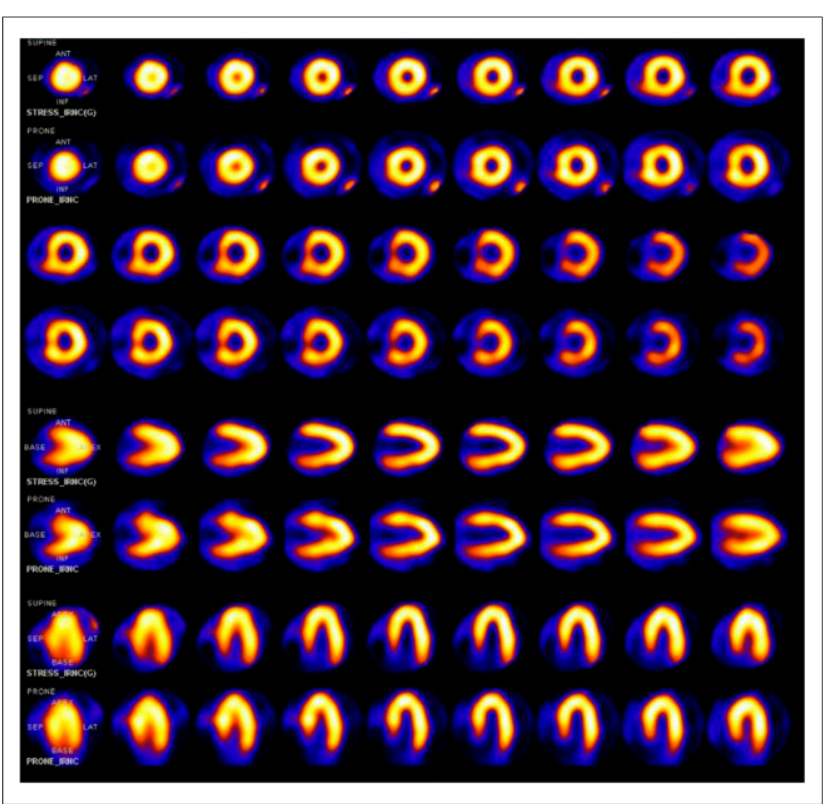

FIGURE 2. Myocardial perfusion images from normal stress-only study performed on high-efficiency camera (patient 89 in this study). Top rows are supine images; bottom rows are prone images. Radiation effective dose for the study was $0.93 \mathrm{mSv}$.

ULD rest imaging on an HE-SPECT camera to standard low-dose A-SPECT imaging in 101 patients at 3 centers. That study found that ULD HE-SPECT imaging was associated with improved image quality, comparable extracardiac activity, and strong correlation with standard A-SPECT in terms of summed rest score, total perfusion deficit, and ejection fraction (9). Duvall et al. (15) imaged 27 patients who received an average of $192 \mathrm{MBq}$ of ${ }^{99 \mathrm{~m}} \mathrm{Tc}$-sestamibi at rest and $562 \mathrm{MBq}$ at stress. They performed both a 15 -min acquisition on an A-SPECT camera and a 5-min acquisition on an HESPECT camera, which was analyzed using both the full dataset and the first $3 \mathrm{~min}$ of data using list mode processing. The authors found significantly higher image quality for the HE-SPECT protocols, with close correlation in total perfusion deficit, ejection fraction, and end-diastolic volume. However, the injected activities used in this study were considerably lower than those typically administered in A-SPECT imaging, raising concerns about the expected quality of imaging in the comparator A-SPECT group. Oddstig et al. (16) performed stress-first HE-SPECT-only imaging in 150 patients who were divided evenly into groups protocoled to receive 4, 3, and $2.5 \mathrm{MBq} / \mathrm{kg}$ of ${ }^{99 \mathrm{~m}} \mathrm{Tc}$-tetrofosmin at stress, with scan acquisition times of 300,400 , and $475 \mathrm{~s}$, respectively. They found no significant difference in image quality between groups. Nakazato et al. (17) performed a simulation study in which low-dose HESPECT was reconstructed retrospectively from high-dose HESPECT. Those authors showed that similar quantitative results for total perfusion deficit and ejection fraction are obtained with counts progressively reduced to less than 1 million in the left ventricular region.

Our study evaluated a stress-first protocol in patients for whom it was deemed clinically appropriate by the attending nuclear cardiologist. Recent work by Duvall et al. (18) has developed a scoring system to predict "unsuccessful" stress-first imaging that requires subsequent rest imaging. Use of this scoring system in conjunction with ULD HE-SPECT imaging may be helpful in developing protocols to further streamline emergency department flow; this hypothesis requires further validation in a cohort receiving ultra-low doses.

Of note, notwithstanding the very low radiation doses achieved, our study used an "on label" $185-\mathrm{MBq}(5-\mathrm{mCi})$ unit dose of ${ }^{99 \mathrm{~m}} \mathrm{Tc}$-tetrofosmin, which is approved by the Food and Drug Administration for activities between 185 and 1,221 MBq (5 and 33 mCi) (19). As per Nuclear Regulatory Commission regulations (title 10 of Code of Federal Regulations part 35.63) (20), this activity determination should be made by direct measurement of radioactivity before medical use, which is the activity reflected in the measured administered activities in Table 2. Although all

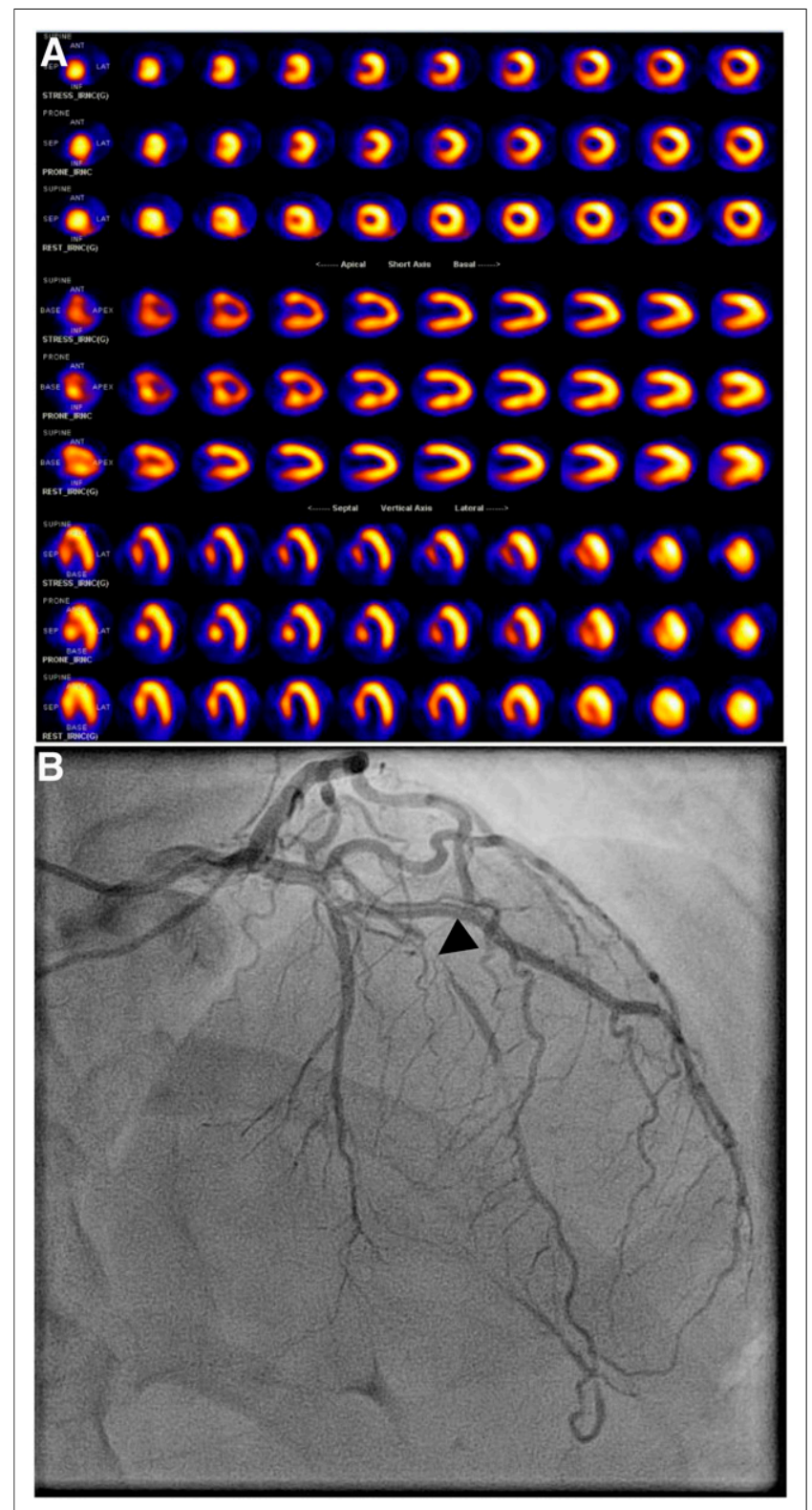

FIGURE 3. Myocardial perfusion images from patient (patient 99 in this study) with anterior and septal ischemia. (A) High-efficiency SPECT image. Radiation effective dose from myocardial perfusion imaging was $0.77 \mathrm{mSv}$ for stress, $2.57 \mathrm{mSv}$ for rest. (B) Invasive coronary angiogram shows $99 \%$ stenosis of left anterior descending artery (arrowhead). 
patients were prescribed an activity of $185 \mathrm{MBq}$ for the stress dose, in 2 patients the actual measured activity was slightly less than $185 \mathrm{MBq}(178 \mathrm{MBq}$ and $184 \mathrm{MBq})$. Our findings are fundamentally unchanged when analyses are repeated excluding these 2 patients. In any event, the Nuclear Regulatory Commission permits administered dosage within $\pm 20 \%$ of the prescribed dosage $(20,21)$, which was the case for all patients in our study.

One challenge posed in ULD MPI is to accurately deliver a desired activity to the patient. ${ }^{99 \mathrm{~m}} \mathrm{Tc}$-based radiopharmaceuticals are "sticky," with some of the radiopharmaceutical adhering to the wall of the syringe and stopcock, and thus the activity received in practice is lower than the measured administered activity. We noted that residual activities of ULD injections of ${ }^{99 \mathrm{~m}} \mathrm{Tc}$-tetrofosmin averaged $42 \mathrm{MBq}$ and ranged from 6.3 to $118 \mathrm{MBq}$. For highdose injections, which typically are around $888-1,332 \mathrm{MBq}$, and even for standard low-dose injections, which typically are 296$444 \mathrm{MBq}$, this variability in residual activity corresponds to a modest fraction of the actual received activity, and thus the administered activity is a reasonable approximation of the received activity; however, for ULD imaging this residual dose can represent most of the administered activity. Because of this phenomenon, it is important when performing ULD imaging to record the residual dose and use it to determine the actual received activity, from which radiation dose can be estimated and acquisition time can be tailored. The residual doses that we observed here were on average lower than those for ${ }^{99 \mathrm{~m}} \mathrm{Tc}$-sestamibi that were observed in the MILLISIEVERT study, in which residual activity for a similar ULD injection averaged $64 \mathrm{MBq}$ and ranged from 6.3 to 144 $\mathrm{MBq}$ (9). Although limited data suggest that tetrofosmin may be

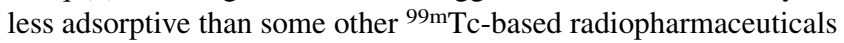
(22), numerous factors influence ${ }^{99 \mathrm{~m}} \mathrm{Tc}$ residual activity, including not just the particular radiopharmaceutical but also the type of syringe and length of time the radiopharmaceutical is in the syringe before injection $(23,24)$. The determinants of residual activity for ULD injections is a subject that requires further study.

Our study is not without limitations. By design, we excluded patients with a BMI of $35 \mathrm{~kg} / \mathrm{m}^{2}$ or higher, for whom data about the suitability of MPI with an HE-SPECT camera are mixed. For example, in a study of 148 obese patients with a mean BMI of 39 $\mathrm{kg} / \mathrm{m}^{2}$, and using activities as low as $185 \mathrm{MBq}$ at stress, Gimelli et al. (25) observed very good or excellent HE-SPECT image quality in all patients and an area under the receiver-operating-characteristic curve of 0.85 for determination of coronary artery disease. However, in another study, Fiechter et al. (26) found image quality less than "good" in a third of patients with a BMI of $35-39.5 \mathrm{~kg} / \mathrm{m}^{2}$ and nondiagnostic image quality in most patients with a BMI of 40 $\mathrm{kg} / \mathrm{m}^{2}$ or higher. Thus, we elected to exclude patients with World Health Organization class II or III obesity for this initial ULD prognostic study. Additionally, our inclusion criteria were limited to patients with chest pain; future research should expand these findings to other patients undergoing MPI. We did not consider alternative testing approaches such as exercise treadmill testing without MPI or coronary CT angiography. A general limitation of ULD imaging using an HE-SPECT camera is that the likelihood of a motion artifact may be increased, particularly in sicker patients with less ability to lie still, because of the 15-min acquisition time and the inability of an HE camera to correct (or even visualize) patient motion during imaging. Nevertheless, in previous work we have shown similar image quality and interpretation between ULD HE-SPECT and standard low-dose A-SPECT imaging (10). Future research should aim to optimize the imaging time for ULD
HE-SPECT MPI. Finally, we did not assess outcomes beyond 1 y. Even so, the primary goal of MPI in patients presenting to the hospital with chest pain is to avoid unsafely discharging those patients with a high short-term risk of major adverse cardiac events. Outcomes beyond $1 \mathrm{y}$ are only of secondary interest, all the more so when the study is of such a low radiation dose that subsequent repeat imaging would not result in a large cumulative radiation burden to the patient.

\section{CONCLUSION}

Our study demonstrates that in patients with chest pain who do not have a high pretest probability of a stress perfusion defect, stress-only SPECT MPI using an HE camera can be performed in more than two thirds of patients with a radiation effective dose averaging $1 \mathrm{mSv}$, in a time averaging less than $2 \mathrm{~h}$, and with an excellent prognosis, evidenced by only $4 \%$ of patients requiring additional cardiac imaging or presenting again to the hospital for chest pain evaluation within $3 \mathrm{mo}$, and no deaths or acute coronary syndromes at $1 \mathrm{y}$.

\section{DISCLOSURE}

The costs of publication of this article were defrayed in part by the payment of page charges. Therefore, and solely to indicate this fact, this article is hereby marked "advertisement" in accordance with 18 USC section 1734 . This study was funded by an investigatorinitiated research grant from GE Healthcare, by the Louis V. Gerstner, Jr., Scholars Program, and by a Herbert Irving Associate Professorship. Andrew Einstein has received support for other research from Philips Healthcare and Spectrum Dynamics. No other potential conflict of interest relevant to this article was reported.

\section{REFERENCES}

1. Niska R, Bhuiya F, Xu J. National Hospital Ambulatory Medical Care Survey: 2007 emergency department summary. Natl Health Stat Report. 2010;6:1-31.

2. Amsterdam EA, Kirk JD, Bluemke DA, et al. Testing of low-risk patients presenting to the emergency department with chest pain: a scientific statement from the American Heart Association. Circulation. 2010;122:1756-1776.

3. Anderson JL, Adams CD, Antman EM, et al. ACC/AHA 2007 guidelines for the management of patients with unstable angina/non-ST-elevation myocardial infarction. J Am Coll Cardiol. 2007;50:e1-e157.

4. European Commission, Directorate-General for the Environment. Referral Guidelines for Imaging: Radiation Protection 118. Luxembourg: Office for Official Publications of the European Communities; 2001.

5. Einstein AJ, Pascual TN, Vitola J, et al. Variation in radiation doses from nuclear cardiology procedures: results from the 66 country INCAPS (IAEA Nuclear Cardiology Protocols) Study [abstract]. J Am Coll Cardiol. 2014;63(suppl): A1226.

6. McNulty EJ, Hung YY, Almers LM, Go AS, Yeh RW. Population trends from 2000-2011 in nuclear myocardial perfusion imaging use. JAMA. 2014;311:12481249.

7. Imbert L, Poussier S, Franken PR, et al. Compared performance of high-sensitivity cameras dedicated to myocardial perfusion SPECT: a comprehensive analysis of phantom and human images. J Nucl Med. 2012;53:1897-1903.

8. Slomka PJ, Berman DS, Germano G. New cardiac cameras: single-photon emission CT and PET. Semin Nucl Med. 2014;44:232-251.

9. Einstein AJ, Blankstein R, Andrews H, et al. Comparison of image quality, myocardial perfusion, and left ventricular function between standard imaging and single-injection ultra-low-dose imaging using a high-efficiency SPECT camera: the MILLISIEVERT study. J Nucl Med. 2014;55:1430-1437.

10. Shoyeb A, Bokhari S, Sullivan J, et al. Value of definitive diagnostic testing in the evaluation of patients presenting to the emergency department with chest pain. Am J Cardiol. 2003;91:1410-1414.

11. Rosner GF, Stone GW, Stant J, et al. Updating an institutional chest pain algorithm: incorporating new evidence on emerging pharmacotherapy. Crit Pathw Cardiol. 2012;11:107-113. 
12. Mattsson S, Johansson L, Leide Svegborn S, et al. Radiation dose to patients from radiopharmaceuticals: a fourth addendum to ICRP publication 53. ICRP website. http://www.icrp.org/docs/Radiation\%20Dose\%20to\%20Patients\%20from \%20Radiopharmaceuticals\%20-\%20A\%20fourth\%20addendum\%20to\%20ICRP\% 20Publication\%2053.pdf. Published May 24, 2013. Revised February 24, 2014. Accessed February 25, 2015

13. Chang SM, Nabi F, Xu J, Raza U, Mahmarian JJ. Normal stress-only versus standard stress/rest myocardial perfusion imaging: similar patient mortality with reduced radiation exposure. J Am Coll Cardiol. 2010;55:221-230.

14. Goto K, Takebayashi H, Kihara Y, et al. Impact of combined supine and prone myocardial perfusion imaging using an ultrafast cardiac gamma camera for detection of inferolateral coronary artery disease. Int J Cardiol. 2014;174:313317.

15. Duvall WL, Croft LB, Ginsberg ES, et al. Reduced isotope dose and imaging time with a high-efficiency CZT SPECT camera. J Nucl Cardiol. 2011;18:847857.

16. Oddstig J, Hedeer F, Jogi J, Carlsson M, Hindorf C, Engblom H. Reduced administered activity, reduced acquisition time, and preserved image quality for the new CZT camera. J Nucl Cardiol. 2013;20:38-44.

17. Nakazato R, Berman DS, Hayes SW, et al. Myocardial perfusion imaging with a solid-state camera: simulation of a very low dose imaging protocol. $\mathrm{J} \mathrm{Nucl}$ Med. 2013;54:373-379.

18. Duvall WL, Baber U, Levine EJ, Croft LB, Henzlova MJ. A model for the prediction of a successful stress-first Tc-99m SPECT MPI. J Nucl Cardiol. 2012;19:1124-1134.
19. Myoview ${ }^{\mathrm{TM}} 30 \mathrm{~mL}$ kit for the preparation of technetium $\mathrm{Tc} 99 \mathrm{~m}$ tetrofosmin for injection. Food and Drug Administration website. http://www.accessdata.fda. gov/drugsatfda_docs/label/2009/020372s022lbl.pdf. Accessed October 29, 2014.

20. $\S 35.63$ determination of dosages of unsealed byproduct material for medical use. U.S. Nuclear Regulatory Commission website. http://www.nrc.gov/readingrm/doc-collections/cfr/part035/part035-0063.html. Accessed October 29, 2014.

21. 10 CFR parts 20, 32, and 35. RIN 3150-AF74. Medical use of byproduct material; final rule. U.S. Nuclear Regulatory Commission website. http://www.nrc.gov/readingrm/doc-collections/commission/secys/2000/secy2000-0118/attachment6.pdf. Accessed October 29, 2014.

22. Bartosch R, Granegger S, Sinzinger H. Adsorption of technetium-99m tetrofosmin and technetium-99m furifosmin on plastic syringes. Eur J Nucl Med. 1998;25:1333-1335.

23. Jansson BA, Goransson MB, Agren BN. Adsorption of some technetium-99m radiopharmaceuticals onto disposable plastic syringes. J Nucl Med Technol. 1998;26:196-199.

24. Swanson TN, Troung DT, Paulsen A, Hruska CB, O'Connor MK. Adsorption of ${ }^{99 \mathrm{~m}} \mathrm{Tc}$-sestamibi onto plastic syringes: evaluation of factors affecting the degree of adsorption and their impact on clinical studies. J Nucl Med Technol. 2013;41: 247-252.

25. Gimelli A, Bottai M, Giorgetti A, Genovesi D, Filidei E, Marzullo P. Evaluation of ischaemia in obese patients: feasibility and accuracy of a low-dose protocol with a cadmium-zinc telluride camera. Eur J Nucl Med Mol Imaging. 2012;39:1254-1261.

26. Fiechter M, Gebhard C, Fuchs TA, et al. Cadmium-zinc-telluride myocardial perfusion imaging in obese patients. J Nucl Med. 2012;53:1401-1406. 